\title{
Sutureless bioprosthesis may increase postoperative atrial fibrillation after aortic valve replacement
}

\author{
Sameer D Thakur ${ }^{1}$, Andrew Cheng ${ }^{2 *}$, Hansraj R Bookun ${ }^{3}$, David Lee ${ }^{2}$, Chin Siew Lee ${ }^{4}$, Xiao Bo Zhang ${ }^{2}$, \\ Shivanand Gangahanumaiah ${ }^{2}$, Marco Moscarelli $i^{5}$, Giacomo Bianchi ${ }^{5}$, Pier A Farneti $i^{5}$, Marco Solinas ${ }^{5}$ \\ From World Society of Cardiothoracic Surgeons 25th Anniversary Congress, Edinburgh \\ Edinburgh, UK. 19-22 September 2015
}

\section{Background/Introduction}

Benefits of sutureless aortic valve replacement (AVR) have been established. Most western centres have reported advantages in reduced cardiopulmonary bypass, cross clamp, ventilation, and postoperative recovery time. It has established a role in moderate to high-risk surgical patients requiring an AVR. However, the incidence of postoperative atrial fibrillation (POAF) in sutureless AVR is less known.

\section{Aims/Objectives}

Investigate the incidence of POAF after sutureless AVR and compare that with the rate of POAF after sutured AVR.

\section{Method}

From January 2001 to January 2015, 1417 AVR cases were performed. Demographic and perioperative data were collected prospectively. A total of 188 patients were excluded from analysis due to a preoperative history or incomplete data. We compared the rate of POAF after sutureless and sutured AVR cases overall and in subgroups divided by access (FS - Full Sternotomy; PS Partial Sternotomy; MT - Mini-Thoracotomy). The incidence of POAF was identified by continuous cardiac monitoring.

Homogeneity of the sample was tested using multivariate regression and Kolmogorov-Smirnov tests, which did not identify any statistically significant confounding variables. Descriptive statistics were used to characterize samples with regards to demographic and perioperative variables.

${ }^{2}$ Department of Cardiothoracic Surgery, University Hospital Geelong, Australia Full list of author information is available at the end of the article

\section{Results}

A total of 1229 patients (604 females) were included in the analysis. The incidence of POAF in sutureless and sutured AVR cases was $35.8 \%$ and $29.5 \%$ respectively. The odds ratio for POAF is 1.33 (95\% CI: 1.03-1.73; $\mathrm{p}=$ 0.031 ) with a sutureless valve. In subgroup analysis, POAF rates in the MT group for sutureless and sutured AVR were $33.1 \%$ and $22.0 \%$ respectively (OR 1.76 95\% CI: 1.19 - 2.59; $\mathrm{p}=0.004)$. POAF rates in the PS group for sutureless and sutured AVR were $50.9 \%$ and $33.3 \%$ respectively (OR 2.07 95\% CI: 1.13-3.80; $\mathrm{p}=0.019$ ). FS had similar rates of POAF in both groups - sutureless $30.4 \%$ and sutured $32.3 \%$.

\section{Discussion/Conclusion}

Sutureless AVR is an important surgical option with proven advantages in moderate to high-risk patients. Prevention of POAF should be considered in patients whom a sutureless AVR is performed.

\section{Authors' details}

'Department of Cardiothoracic Surgery, Royal Hobart Hospital, Australia. 2Department of Cardiothoracic Surgery, University Hospital Geelong, Australia. ${ }^{3}$ Department of Vascular Surgery, University Hospital Geelong. ${ }^{4}$ Cardiology Research Unit, University Hospital Geelong, Australia.

${ }^{5}$ Cardiothoracic Department, Fondazione Toscana G. Monasterio, Massa, Italy.

Published: 16 December 2015

doi:10.1186/1749-8090-10-S1-A26

Cite this article as: Thakur et al.: Sutureless bioprosthesis may increase postoperative atrial fibrillation after aortic valve replacement. Journal of Cardiothoracic Surgery 2015 10(Suppl 1):A26. 